\title{
Machine learning-based Time Series Models for Effective CO2 Emission prediction in India
}

\section{Sunil Kumar Singh ( $\nabla$ sksingh@mgcub.ac.in )}

MGCUB: Mahatma Gandhi Central University https://orcid.org/0000-0001-8954-6648

\section{Surbhi Kumari}

Mahatma Gandhi Central University

\section{Research Article}

Keywords: Time Series Forecasting, Linear Regression, Random Forest Regressor, Air Pollution, CO2 Emissions, Holt-Winter, LSTM

Posted Date: February 7th, 2022

DOI: https://doi.org/10.21203/rs.3.rs-1265771/v1

License: (c) (1) This work is licensed under a Creative Commons Attribution 4.0 International License. Read Full License 


\title{
Machine learning-based Time Series Models for Effective $\mathrm{CO}_{2}$ Emission prediction in India
}

\author{
Surbhi Kumari ${ }^{1}$, Sunil Kumar Singh ${ }^{2 *}$ \\ ${ }^{1,2}$ Dept. of Computer Science and Information Technology \\ Mahatma Gandhi Central University, Motihari, Bihar, India \\ *Email: sksingh@mgcub.ac.in; sunilsingh.jnu@gmail.com \\ *corresponding author
}

\begin{abstract}
As per the report of datacommons.org, $\mathrm{CO}_{2}$ emission in India is 1.80 metric tons per capita, which is very harmful for living beings. So, this paper presents India's detrimental $\mathrm{CO}_{2}$ emission effect with the prediction of $\mathrm{CO}_{2}$ emission for the next ten years based on univariate time series data. We used three statistical models i.e., AutoRegressive Integrated Moving Average (ARIMA) model, Seasonal AutoRegressive Integrated Moving Average with eXogenous factors (SARIMAX) model, and Holt-Winter model, two machine learning models, i.e., Linear Regression and Random Forest model and one deep learning model, i.e. Long Short Term Memory (LSTM) model. The performance analysis shows that LSTM, SARIMAX, and Holt-Winter methods are accurate models and have the Least Mean Squared Error(MSE), Root Mean Square Error (RMSE), and Median Absolute Error (MedAE) for this kind of univariate data distribution.
\end{abstract}

Keyword - Time Series Forecasting, Linear Regression, Random Forest Regressor, Air Pollution, $\mathrm{CO}_{2}$ Emissions, HoltWinter, LSTM

\section{Introduction}

According to Ministry of Statistics and Programme Implementation, UN (World Population Prospects 2019) the current population of India is 1,400,517,328 as of January 2022 based on interpolation of the latest United Nations data which is the second-largest, just falling behind China and standing at second in the world. It would catch China and even surpass it shortly if it continues to grow at the same rate. With this, environmental consequences which may arise are many but $\mathrm{CO}_{2}$ emission remains the topmost concern because of the problems which ensue due to its increased rate (Bonga \& Chirowa, 2014). According to UN data, India's $\mathrm{CO}_{2}$ emission rose faster than the world average of $0.7 \%$. Increased $\mathrm{CO}_{2}$ will accentuate the world's food and water crisis and increase the incidences of natural disasters.

The increased flooding, landslide, cloud bursts, etc. are already evident and would further increase if we continue to go the same way. Forecasting $\mathrm{CO}_{2}$ emissions are also one important key to creating awareness among the public on solving environmental problems(Abdullah \& Pauzi, 2015). So, the proposed work has carefully examined the three statistical models, two machine learning models, and a deep learning model for time series $\mathrm{CO}_{2}$ emission forecasting and also did performance analysis based on nine metrics to get the best performing model for the emission of $\mathrm{CO}_{2}$ till 2030. By carefully examining the outcomes of different models, we found some models to be fairly viable forecasts. The data used is of the past 40 years, which plots the increase in $\mathrm{CO}_{2}$ emission against time.

The forecast we have come up with will help to understand our pace of emission and further correction we need to do to keep the temperature rise under control. This would help future policy actions necessary to develop India's Nationally Determined Contributions Pledge which India has taken at Paris agreement. This paper has also focused on finding which time series models i.e. statistical models, machine learning models, and deep learning models, are best suited for this kind of $\mathrm{CO}_{2}$ emissions data.

And it gives an idea for future papers which could use these models and refine the future forecast by taking into account other exogenous variables such as increasing population, technology advancement, and several other future technologies and policy actions that may positively or negatively affect the $\mathrm{CO}_{2}$ emission. This paper has made a comparative analysis of different models and their accuracy in forecasting $\mathrm{CO}_{2}$ emission, which would be helpful for future researchers to ascertain the forecasting models suitable for the purpose. 
Many works have been done in time series data using machine learning, deep learning, and statistical model. Let us know about some of them. In the first paper (Masini et al., 2021), the author survey the most recent advances in supervised machine learning (ML) and high dimensional models by considering both linear and non-linear methods for time-series forecasting and considering ensemble and hybrid models by combining ingredients from different alternatives. They also apply time series forecasting in the economic and financial fields. In (Crespo Cuaresma et al., 2004), the author studies the forecasting abilities of a battery of univariate models and includes AutoRegressive (AR) models.

In (Elsworth \& Güttel, 2020), the author proposed an RNN model with a dimension reducing symbolic representation to deal with the sensitivity of hyperparameters in any time series model and solves the limitation of other models, that is the initialization of random weights. Also, his model is faster without affecting the performance of forecasting. Also, in (Zuo et al., 2020) author collected the $\mathrm{CO} 2$ emission data from the different provinces of China and proposed a model named LSTM-STRIPAT, an integrated model to predict emission in 2020, and in (Amarpuri et al., 2019)the research is aimed at predicting the $\mathrm{CO} 2$ levels in the year 2020 to make the Government of India understand the challenges. A deep learning hybrid model of Convolution Neural Network and Long Short-Term Memory Network (CNN-LSTM) is used as a forecasting model.

A prediction based work was done in (R. Kumar et al., 2020), in which they have collected the data from Delhi and National Capital Region (NCE), India to predict the Air quality index. For the same, they have applied the machine learning models and measured the performances in terms of MAE, MSE, RMSE, and MAPE metrics. Similar work was done in (S. Kumar et al., 2020) to measure the PM2.5 pollutant particles in the Delhi atmosphere. In this work, Extra-Trees regression and AdaBoost based regression model is applied to predict PM2.5 concentrations effectively. They have also considered additional atmospheric and surface factors such as wind speed, atmospheric temperature, pressure, etc.

The work (Ahmed et al., 2010) applied various eight machine learning models on famous M3 time series competition data and compared them. The models' multilayer perceptron and the Gaussian process regression performed the best. In (Nyoni \& Bonga, 2019) author used the Box- Jenkins ARIMA approach on time series data of $\mathrm{CO}_{2}$ emission in India from 1960 to 2017 , and based on forecast, they suggested five policy prescriptions to improve the environmental conditions.

ARIMA model has also been used in the analysis of air pollutants and to predict them based on historical data in (Gopu et al., 2021) and also said that this is an efficient way by which we can find out the values of the pollutants when exceeding the limits prescribed by the World Health Organization (WHO). SARIMA is an ARIMA capable of dealing with the seasonality of the dataset, and SARIMAX is SARIMA with $\mathrm{X}$ factor, which is nothing but exogenous factors that affect the data. The paper (Nontapa et al., 2020) analysed and forecasted using the SARIMA and SARIMAX model with decomposition method and compared them on evaluation metric MAPE where SARIMAX with decomposition model outperforms traditional ones. There is information regarding time series forecasting and limitation with the SARIMAX model in paper (Özmen, 2021)with the application of retail store data. In this work of CO2 emission, a Random Forest regressor has been used for forecasting. In the paper (Fang et al., 2020) author proposed an optimal random forest model for the accurate prediction of an infectious diarrhoea epidemic, considering meteorological factors. He compared his proposed model to ARIMA and ARIMAX, and the RF model outperforms with MAPE of approximately $20 \%$ to the ARIMA model with MAPE reaching 30\%. In the paper (Lepore et al., 2017) author describes that with the introduction of the EU's new $\mathrm{CO} 2$ emissions regulation, ships' operators are required to implement systems that will monitor and report their $\mathrm{CO} 2$ emissions. These systems will help them make informed decisions regarding their operations.Due to the complexity of the navigation information that ships provide, there is no widely available standard method or solution that can be used in real environments. This paper presents an extensive analysis of the various regression techniques that can be used to exploit the navigation information that ships provide.The study is made based on the data collected by the Grimaldi Group's Ro-Pax cruise ship during its operation. It aims to identify possible methods and models that can be used to analyze the ship's $\mathrm{CO} 2$ emissions and develop a predictive model.

There is a project which aims to develop models and artificial neural networks to predict the energy consumption of office buildings in Chile and CO2 emissions(Pino-Mejías et al., 2017). Eight fundamental variables have been used to analyze the design parameters of commercial air-cooled cooling and heating systems. The results of the study show that the linear regression models with higher accuracy and better performance are those with the least amount of predictive errors. It is expected that the models will help estimate the energy savings that different design concepts would produce during the 
construction phases.This procedure was developed to generate training data for ANN using a statistical method. The resulting database contains case files that are representative of the office buildings. The statistical models that rely on the multi-perceptron method are more accurate than those that rely on the standard linear regression model. They can reproduce the results of ISO 17000:2008 with high accuracy. This study aimed to develop an ANN model that could predict office buildings' energy consumption and greenhouse gas emissions in Santiago, Chile. The model was tested on a non-standard set of factors. This method will help designers and developers to develop more energy-efficient and accurate methods for calculating greenhouse gas and energy consumption. The framework proposed in this study can be used to develop energyefficient building standards that are realistic and sustainable. A paper (Wang et al., 2020) is the study, that the data on carbon emissions be accurate for developing effective carbon mitigation strategies. For China, the US, and India, the data on their carbon emissions are different.The US carbon emissions data shows volatile growth and decline. This paper Author proposes a method to improve the accuracy of the data by developing a combination of the ARIMA and the MNGM models. This method could reduce the residual error of the model MNGM by applying ARIMA and BPNN. It can also decrease the forecasting error of the model. As for the US' carbon emissions, it is expected to keep a downward trend during the next couple of decades.

Meanwhile, China and India's carbon emissions will keep growing. The proposed two forecasting techniques have shown that they can improve the forecasting models' accuracy by correcting previous errors. They also exhibited sound performance for the three different types of data.There is a statistical Model called Holt-Winter(Chatfield, 1978) is there for dealing with trends and seasonal variation. According to this paper, some available idiosyncratic modifications can improve the Holt-Winter's Forecasts. There is an argument that a fairer comparison would be that between Box-Jenkins and a non-automatic version of Holt-Winters. There is a suggested method for predicting and estimating trends in specific datasets. If there are multiple trends in data and the forecasting model dimensions increase, this trend estimation becomes a more tedious task. So paper (Sbrana, 2021) has a closed-form result for simple prediction and estimation of the multivariate smooth-trend model, a state-space representation of Holt-Winters celebrated recursions.

\section{The Dataset}

We have $\mathrm{CO}_{2}$ and greenhouse gas emission data ${ }^{1}$ of 40 years in this work. We have collected the data from the year 1980 to 2019 by using the CAIT data source. The CAIT dataset is the most comprehensive and includes all the sectors along with gases. Greenhouse gas emission data indicates that $60 \%$ of the GHG emissions are from the top 10 emitting countries. In this work, we have used the univariate time series data of India, having an increasing $\mathrm{CO}_{2}$ emission trend.

Before splitting the data into training and testing samples, the data preprocessing is done. Test data is used to evaluate the performance of the models, and better performing models are used for forecasting the $\mathrm{CO}_{2}$ emissions.

\section{Proposed Model}

As we talked about in an earlier section that $\mathrm{CO} 2$ emission in India is growing very faster and is very dangerous for the environment and ultimately to all living beings, the reduction of this emission should be the highest priority for government and industries. An accurate forecast of emissions would direct help in policy-making and implementing them. So for this purpose, we have a CO2 emission's time series data with some attributes for the last 40 years, from 1980 to 2019 . The requirement for this work was a univariate dataset, so it needed some data pre-processing and also data cleaning to deal with not available values in the dataset. The dataset contains two columns, one for years and the second for Co2 emission in metric tons.

Based on these previous data, we have used many time series models to forecast India's ten years CO2 emission. The dataset has an increasing trend and is also stationary and for this particular kind of dataset, we have used three statistical models say ARIMA, SARIMAX, and Holt-Winter model, two machine learning models i.e. Linear Regression model and Random Forest regression model, and in last a deep learning model LSTM (S. Kumar et al., 2021).before using dataset in these models we need to split them into two parts i.e training data and testing data to train and test the models.

Different models have different ways to deal and work with data. Let discuss these six time series models briefly :

\footnotetext{
${ }^{1}$ https://www.climatewatchdata.org/ghg-emissions?end_year=2018\&gases=all-ghg\%2Cco2\&start_year=1990
} 
Auto-Regressive Integrated Moving Average (ARIMA): We start with a basic stationary model like AR and MA in time series analysis. But to handle non-stationary models, we go with ARIMA. MA, AR, and ARMA are specific cases of the ARIMA model.

A model that uses the dependency between an observation and a residual error from a moving average model applied to lagged observations. ARIMA is an acronym that stands for Auto-Regressive Integrated Moving Average. Specifically,AR (Autoregression), which is a model that uses the dependent relationship between an observation and some number of lagged observations, I (Integrated) is the use of differencing of raw observations in order to make the time series stationary and MA (Moving Average).

A standard notation is used of $\operatorname{ARIMA}(p, d, q)$ where the parameters are substituted with integer values to quickly indicate the specific ARIMA model being used. Where $p$ is the number of lag observations included in the model, also called the lag order, $d$ is the number of times that the raw observations are differenced, also called the degree of differencing and $q$ is the size of the moving average window, also called the order of moving average. In the work, we have applied ARIMA $(1,2,1)$ to predict the $\mathrm{CO}_{2}$ emissions(Wellington, 2019).

Seasonal Autoregressive Integrated Moving Average with an Exogenous Variable (SARIMAX): Before knowing about SARIMAX, we will try to understand SARIMA. SARIMA is Seasonal Autoregressive Integrated Moving Average in which along with the trend there is seasonality as well. This is ARIMA, but having the effect of seasonality is something with those time series that have both trends and seasonality. Now, we have to understand the X factor of SARIMAX, which is an Exogenous Variable that means there is some external factor that is impacting it. It is saying that there are some factors outside of the overall factor that we are studying (Özmen, 2021).

Holt-Winter's Model: The Holt Winter model is a traditional model dealing with time-series data behaviour. These behaviors here mean the average value, the trend increasing or decreasing trend, and the seasonality which is nothing but the repetitive pattern in a cycle. Based on the seasonal component of data, this model has two variations. The first is the Additive Holt-Winter Model, and the second is the Multiplicative Holt-Winter. In this work, the multiplicative model has been used to forecast the next 10 years' data. The multiplicative Holt-Winters is described as a method that calculates the value of the level, trend, and seasonal adjustment that are exponentially smooth. Since this method is best suited for data with increasing trends and seasonality, this paper considers this model for $\mathrm{CO}_{2}$ emission forecasting (Chatfield, 1978).

Linear Regression: Linear regression is a Machine learning model to solve regression problems. This model solves the problem by assuming a linear relationship between the given input attributes and the output as shown in equation (1).

Target Output $=$ Input $_{1} *$ weight $_{1}+$ Input $_{2} *$ weight $_{2} \ldots \ldots \ldots \ldots \ldots$ Input $_{n} *$ weight $_{n}+$ Bias

Here $n$ is a total number of attribute/input features. Weights are also called regression coefficients learned while training the model based on train data. The bias here is nothing but the intercept as linear regression is based on the linear equation. Here time series forecasting is also a regression problem where inputs are previous data. In this work, $\mathrm{CO}_{2}$ emission data is univariate data, In this paper $\mathrm{CO}_{2}$ emission data is univariate data, and for each year ,prediction the last three year's data considered as 3 input features for the whole training, testing, and forecasting.Like $\mathrm{CO}_{2}$ emission of year 1980, 1981, 1982 are used to predict the emission in the year 1983 and so on (Huang \& Hsieh, 2020).

Random Forest Regressor: The Random Forest model is a supervised technique for both classification regression and nonlinear problems. This method uses the ensemble learning method for regression and is a bagging technique as it uses individual decision trees together to give better results. This can also be used in time series forecasting, but results are not sure to be expected. In this model, data should be in a proper way before fitting into the model. Data splitting has been done as we have univariate $\mathrm{CO}_{2}$ emission data. One of the advantages of the random forest model is that it handles the missing values and maintains accuracy (Fang et al., 2020).

Long Short-Term Memory (LSTM): LSTM is a deep learning model which is based on a recurrent neural network (RNN). This model is considered to be the best model for processing, classifying, and forecasting time series data. Traditional RNN has the problem of exploding and vanishing gradient descent, so LSTMs were developed to deal with it. For univariate time series forecasting, there is a number of variations of LSTM. Some of them are:Vanilla LSTM, Stacked LSTM, Bidirectional LSTM,CNN LSTM and ConvLSTM (Elsworth \& Güttel, 2020). 
The flowchart shown in the figure 1, presents the framework of the proposed model. In which, it can be seen that we have applied the time-series and machine learning models as stated above.

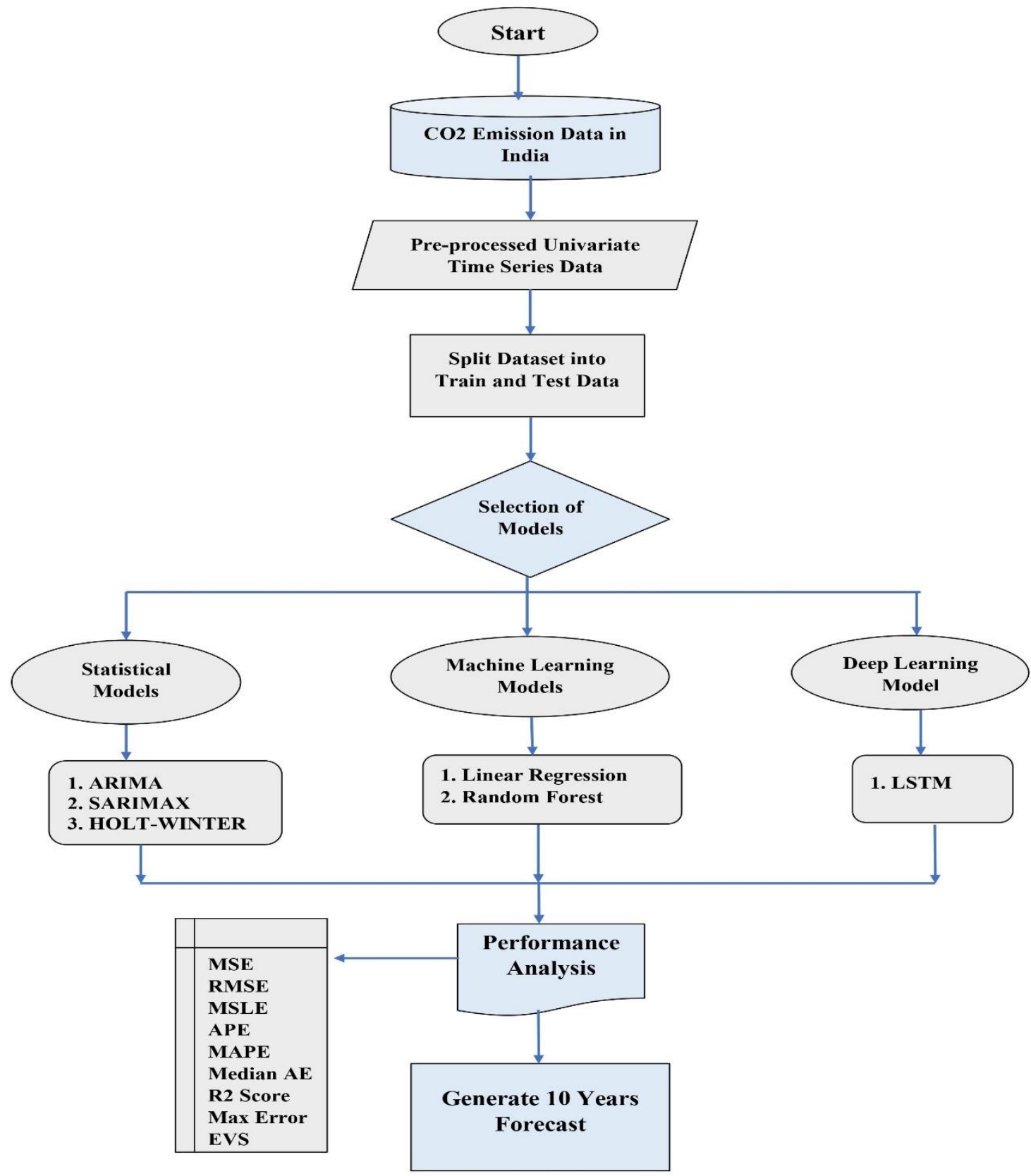

Figure 1: Proposed framework for CO2 Emission forecasting

190 In this framework, we have applied Statistical, Machine learning and deep learning models to forecast the $\mathrm{CO}_{2}$ emissions.

191 For the same, we have used nine performance metrics to analyze the effectiveness of the applied models. Based on the 192 efficiancy of the models, best performing models are used to forecast the $\mathrm{CO}_{2}$ emissions for the next ten years i.e. from 1932020 to 2030. 
Before applying the models, we have pre-processed the data to cope up with the missing values. We have split the preprocessed data into two parts; training and testing. Performance of the models on test data shows the efficacy; and accordingly, it is used to forecast the $\mathrm{CO}_{2}$ emission for the next 10 years till 2030.

\section{Performance metrics}

In this section, we have discussed the performance metrics used to determine the effectiveness of the models. These models forecast $\mathrm{CO}_{2}$ emission, and forecasting, which is a kind of regression problem. There are many evaluation metrics in general; we have used herer nine to evaluate the models' effectiveness. Before using all these metrics, we must know about the residual error, i.e. $(y-\hat{y})$. Here $y$ and $\hat{y}$ Indicates the actual and predicted values. The performance metrics used to evaluate the models are as follows.

Mean Squared Error (MSE): In MSE, first calculate the squares of the residual error as defined above for each data point and then calculate the average of that(R. Kumar et al., 2020). The formula for Mean squared error is shown in equation (2).

$$
M S E=\frac{1}{n} \sum_{i=1}^{n}\left(y_{i}-\hat{y}_{i}\right)^{2}
$$

Where, $y_{i}$ and $\hat{y}_{i}$ indicates the actual and predicted values respectively. $n$ indicates the number of data points.

Root Mean Squared Error (RMSE): It is the same as MSE, the only addition is a square root sign to it(R. Kumar et al., 2020). The formula for Mean absolute error is represented in equation (3).

$$
R M S E=\sqrt{\sum_{i=1}^{n} \frac{\left(\hat{y}_{i}-y_{i}\right)^{2}}{n}}
$$

Mean squared Log Error: In MSLE,the residual error is calculated with the logarithm of the original and predicated value.It is an extension on Mean Squared Error (MSE) mainly used when predictions have large deviations.The formula for Mean absolute log error is represented in equation (4).

$$
M S L E=\frac{1}{n} \sum_{i=1}^{n}\left(\log \left(y_{i}\right)-\log \left(\hat{y}_{i}\right)\right)^{2}
$$

Mean Absolute Error: MAE is the sum of the absolute residual error. That means it does not matter about negative and positive(R. Kumar et al., 2020)i. The formula for Mean absolute error is indicated in equation (5).

$$
M A E=\frac{\sum_{i=1}^{n}\left|\hat{y}_{i}-y_{i}\right|}{n}
$$

Mean Absolute Percentage Error: With the addition of percentage to MAE, MAPE is defined(R. Kumar et al., 2020). The formula for Mean absolute percentage error is shown in equation (6).

$$
M A P E=\frac{100 \%}{n} \sum_{i=1}^{n}\left|\frac{y_{i}-\hat{y}_{i}}{y_{i}}\right|
$$

Median Absolute Error: The median absolute error is robust to outliers, making it intresting and capable enough to deal with impacts of outliers on whole predication (Yin \& Xie, 2021). The formula for Median absolute error is represented by

$$
\operatorname{MedAE}(y, \hat{y})=\operatorname{median}\left(\left|y_{1}-\hat{y}_{1}\right|, \ldots \ldots \ldots\left|y_{n}-\hat{y}_{n}\right|\right)
$$

Max Error: The max error metric calculates the maximum residual error. The formula for Max error is shown in equation (8).

228 R2 Score Error: The formula for R2 Square error is also having it significance to measure the effectiveness of the models 229 (Shaikh et al., 2021), it is defined as shown in equation (9). 


$$
R 2=1-\frac{\sum_{i=1}^{n}\left(\hat{y}_{i}-y_{i}\right)^{2}}{\sum_{i=1}^{n}\left(y_{i}-\bar{y}_{i}\right)^{2}}
$$

Explained Variance Score Error: The Explained variance score error is calculated to measure the proportion of the variability of the predictions of the applied machine learning models. Variance is a measure of how far observed values differ from the average of predicted values, i.e., their difference from the predicted value mean (dos Santos et al., 2021). It can be seen as indicated in equation (10).

$\operatorname{EVS}(y, \hat{y})=1-\frac{\operatorname{var}(y-\hat{y})}{\operatorname{var}(y)}$

\section{Performance Analysis}

The performance of the proposed framework is analyzed by writing a program in a python 3.6 programming environment. We have also used Keras and Tensor flow libraries to implement the machine learning and deep learning-based models. The applied models for predictions are analyzed as follows.

\subsection{Experiment: Performance observation of the models}

We have applied the models on $\mathrm{CO}_{2}$ emission dataset, after splitting the dataset into training and test samples. Trained models are applied on the test dataset to observe the models' performances. Table 1, shows the performances of the applied models, here, in Table 1, we can see that MSLE value for ARIMA model is not available because it can not be applied.

Table 1: The error rate of models on given $\mathrm{CO} 2$ emission dataset

\begin{tabular}{|l|l|l|l|l|l|l|}
\hline & ARIMA & SARIMAX & $\begin{array}{l}\text { Holt- } \\
\text { Winter }\end{array}$ & $\begin{array}{l}\text { Random Forest } \\
\text { Regressor }\end{array}$ & $\begin{array}{l}\text { Linear } \\
\text { Regression }\end{array}$ & LSTM \\
\hline MSE & 1654252.446 & 4654.124 & 20771.376 & 975659.575 & 58016.926 & 3676.646 \\
\hline RMSE & 1286.177 & 68.221 & 144.123 & 987.755 & 240.867 & 60.635 \\
\hline MSLE & NA & 0.830 & 0.004 & 0.787 & 0.0180 & 0.001 \\
\hline MAE & 1099.065 & 44.155 & 113.441 & 771.631 & 196.571 & 45.524 \\
\hline MAPE & 98.969 & 6.554 & 5.043 & 137.238 & 12.023 & 3.101 \\
\hline MEDIAN AE & 898.047 & 30.823 & 115.816 & 550.206 & 155.177 & 28.898 \\
\hline MAX ERROR & 2639.0572 & 314.016 & 243.078 & 2057.741 & 557.092 & 135.933 \\
\hline R2 SCORE & -2.744 & 0.989 & 0.737 & $-7.55 \mathrm{E}+31$ & 0.894 & 0.990 \\
\hline EVS & -0.010 & 0.990 & 0.895 & $-2.94 \mathrm{E}+31$ & 0.965 & 0.990 \\
\hline
\end{tabular}

Further, we have also plotted the bar graph of the applied models to observe the relative performances, as shown in figure 2. Observations from fig-2(a), 2(b), and 2(f), it can be seen that LSTM, SARIMAX, and Holt-Winter models have lowest MSE, RMSE, and MAE values in comparison to other models.

Similarly, thery are having lower MAPE and Max Error values in decreasing order of w.r.t. models SARIMAX, HotWinter, and LSTM.

Observations form fig-2(d) SARIMAX, and LSTM have the lowest MAE values. Similarly, when we look to the R2 Score Error and EVS Error in fig- 2(g) and fig-2(i) the models Holt-Winter, Linear Regression, and SARIMAX have the least error value in increasing order. In last we have remaining MSLE referring fig -2(c) LSTM, Holt-Winter, and Linear 
Regression have the least error value in ascending order. Also, ARIMA is not participating in it negative predictions, which 255 MSLE cannot handle.
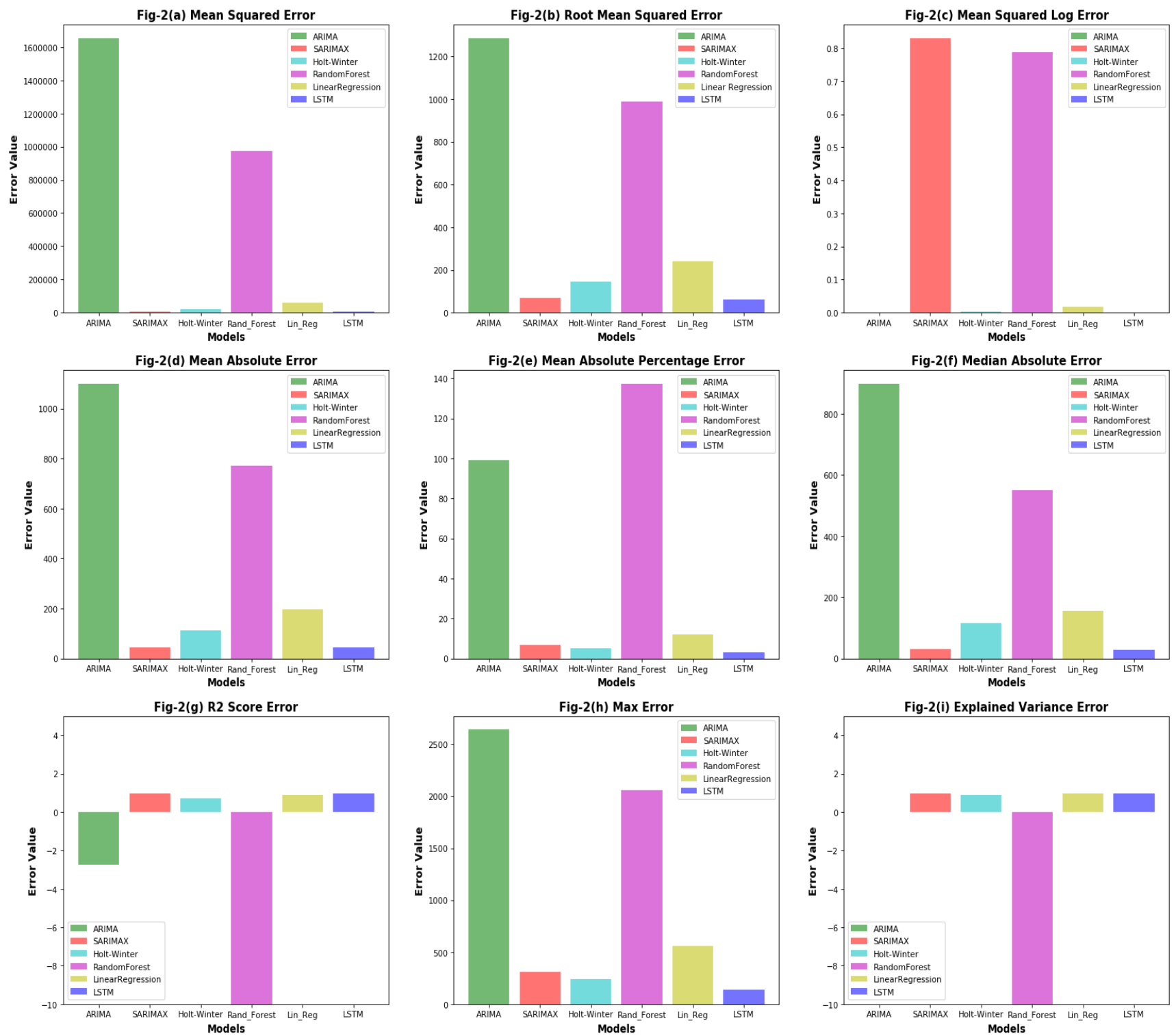

Figure 2: Comparative error plot w.r.t models

Overall observation from figure 2, can be drawn that LSTM, SARIMAX, and Hot-Winter are better performing models for time series forecasting data.

These three models can be applied for effective $\mathrm{CO}_{2}$ emissions predictions. When looking at all nine performance metric values, we can say that LSTM is on the best performing model.

\subsection{Experiment: 10 years forecasting of $\mathrm{CO}_{2}$ emissions}

In this section of the experiment, we have applied the models to forecast the $\mathrm{CO}_{2}$ emissions for next 10 years i.e. from 2020 to 2030. Table 2, shows the summarized results of forecasted values of the models. 
Table 2: 10 Years Forecast of All Models

\begin{tabular}{|l|l|l|l|l|l|l|}
\hline Year & $\begin{array}{l}\text { ARIMA } \\
\text { Model }\end{array}$ & $\begin{array}{l}\text { SARIMAX } \\
\text { Model }\end{array}$ & $\begin{array}{l}\text { Linear } \\
\text { Regression }\end{array}$ & $\begin{array}{l}\text { Random forest } \\
\text { Regressor }\end{array}$ & LSTM Model & $\begin{array}{l}\text { Holt-Winter } \\
\text { Model }\end{array}$ \\
\hline $\mathbf{2 0 2 0}$ & -66.235 & 2744.336 & 3313.565 & 562.259 & 2798.554 & 2903.261 \\
\hline $\mathbf{2 0 2 1}$ & -0.280 & 2932.130 & 3771.178 & 562.259 & 2921.785 & 3037.139 \\
\hline $\mathbf{2 0 2 2}$ & 2.780 & 3060.027 & 4582.197 & 562.259 & 3102.743 & 3110.804 \\
\hline $\mathbf{2 0 2 3}$ & 2.921 & 3168.172 & 5388.920 & 562.259 & 3302.388 & 3281.392 \\
\hline $\mathbf{2 0 2 4}$ & 2.921 & 3355.428 & 6460.649 & 562.259 & 3477.855 & 3394.070 \\
\hline $\mathbf{2 0 2 5}$ & 2.921 & 3429.468 & 7679.896 & 562.259 & 3691.419 & 3529.761 \\
\hline $\mathbf{2 0 2 6}$ & 2.921 & 3646.887 & 9183.267 & 562.259 & 3912.318 & 3595.711 \\
\hline $\mathbf{2 0 2 7}$ & 2.921 & 3655.132 & 10959.754 & 562.259 & 4135.085 & 3773.705 \\
\hline $\mathbf{2 0 2 8}$ & 2.921 & 3675.008 & 13106.276 & 562.259 & 4383.752 & 3884.879 \\
\hline $\mathbf{2 0 2 9}$ & 2.921 & 3816.568 & 15669.673 & 562.259 & 4641.486 & 4022.382 \\
\hline $\mathbf{2 0 3 0}$ & 2.921 & 3969.219 & 18749.809 & 562.259 & 4912.014 & 4080.618 \\
\hline
\end{tabular}

270 Further, we have shown the graphical representation of the models to observe the performance w.r.t. actual and predicted 271 values from 1980 to 2020. Then further forecasted for next ten years.

272 In this sections, we have plotted the graph for actual $\mathrm{CO}_{2}$ emissions for the entire dataset and predicted the values from 2732010 to 2020 by applying the aforesaid models. Further, we have forecasted the values from 2020 to 2030.

\subsubsection{ARIMA's Forecasting}

From figure 3, it can be observed that ARIMA predicted values are too far from the actual $\mathrm{CO}_{2}$ emissions. Therefore, it

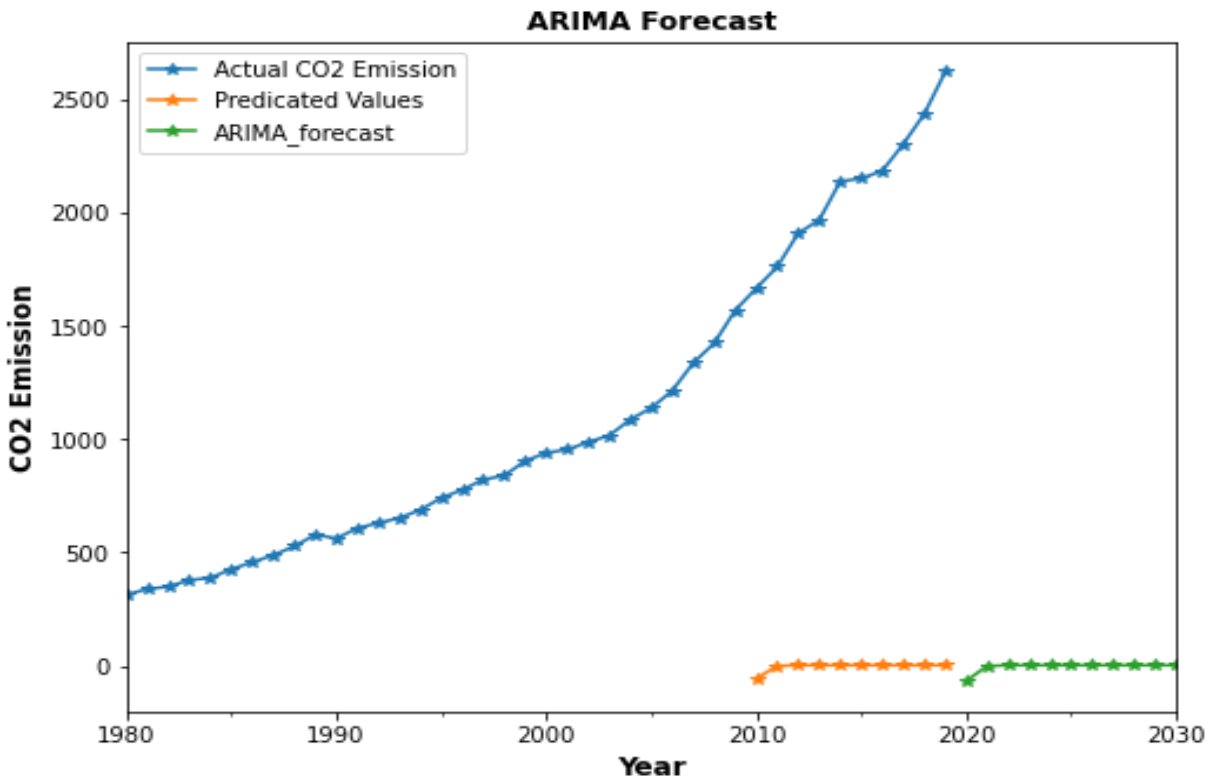




\subsubsection{SARIMAX Forecasting}

282 SARIMAX forecasting is almost in the same pattern as the actual $\mathrm{CO}_{2}$ emission can be seen from figure 4. It can be used 283 as one of the appropriate models for forecasting the $\mathrm{CO}_{2}$ emissions.

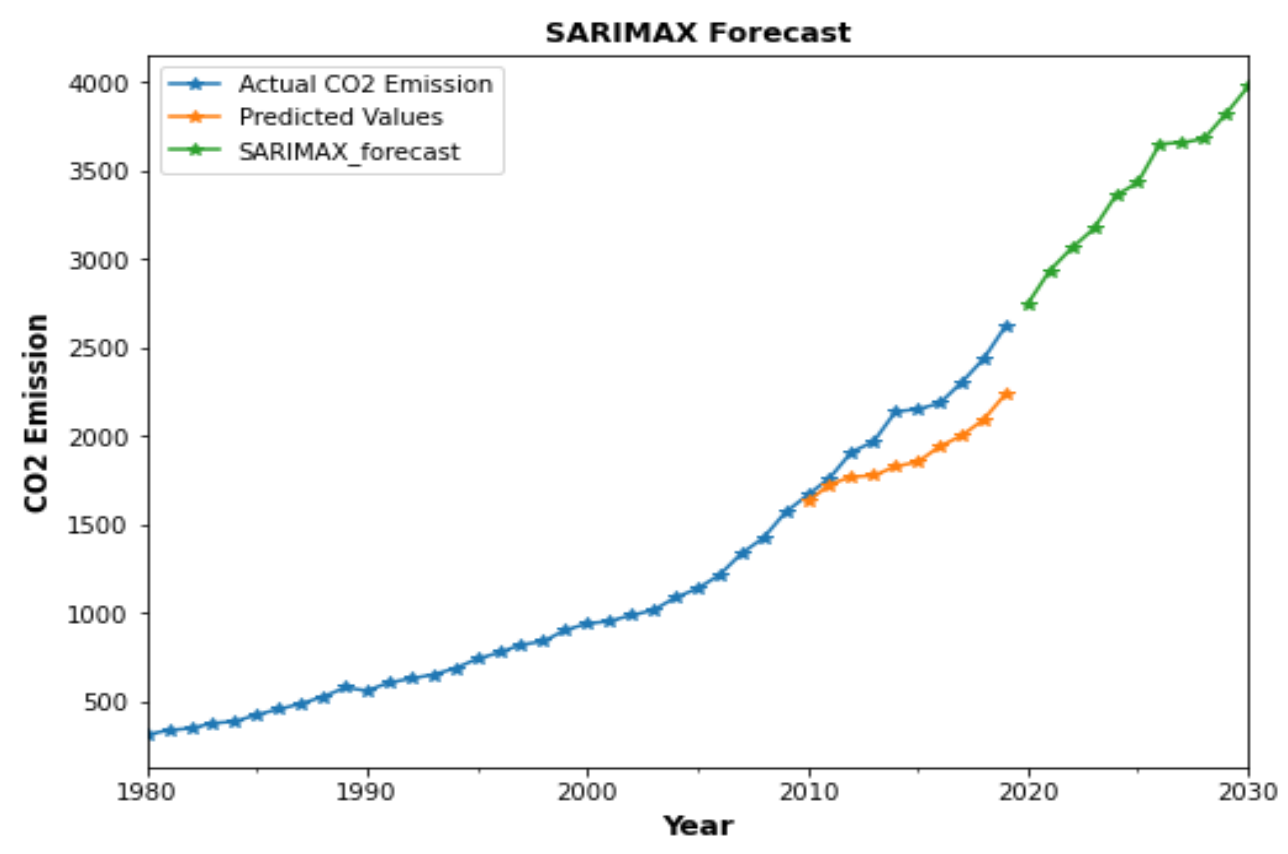

Figure 4. SARIMAX forecasting vs. Actual $\mathrm{CO}_{2}$ emission

\subsubsection{Linear Regression Forecasting}

Figure 5, shows that $\mathrm{CO}_{2}$ predicted values by linear regression and actual emission, which is quite similar. Forecasting values are also in the same pattern as we can see the actual $\mathrm{CO}_{2}$ emissions.

But because of performance metric values of linear regression on test data, its hard to rely on forecasting.

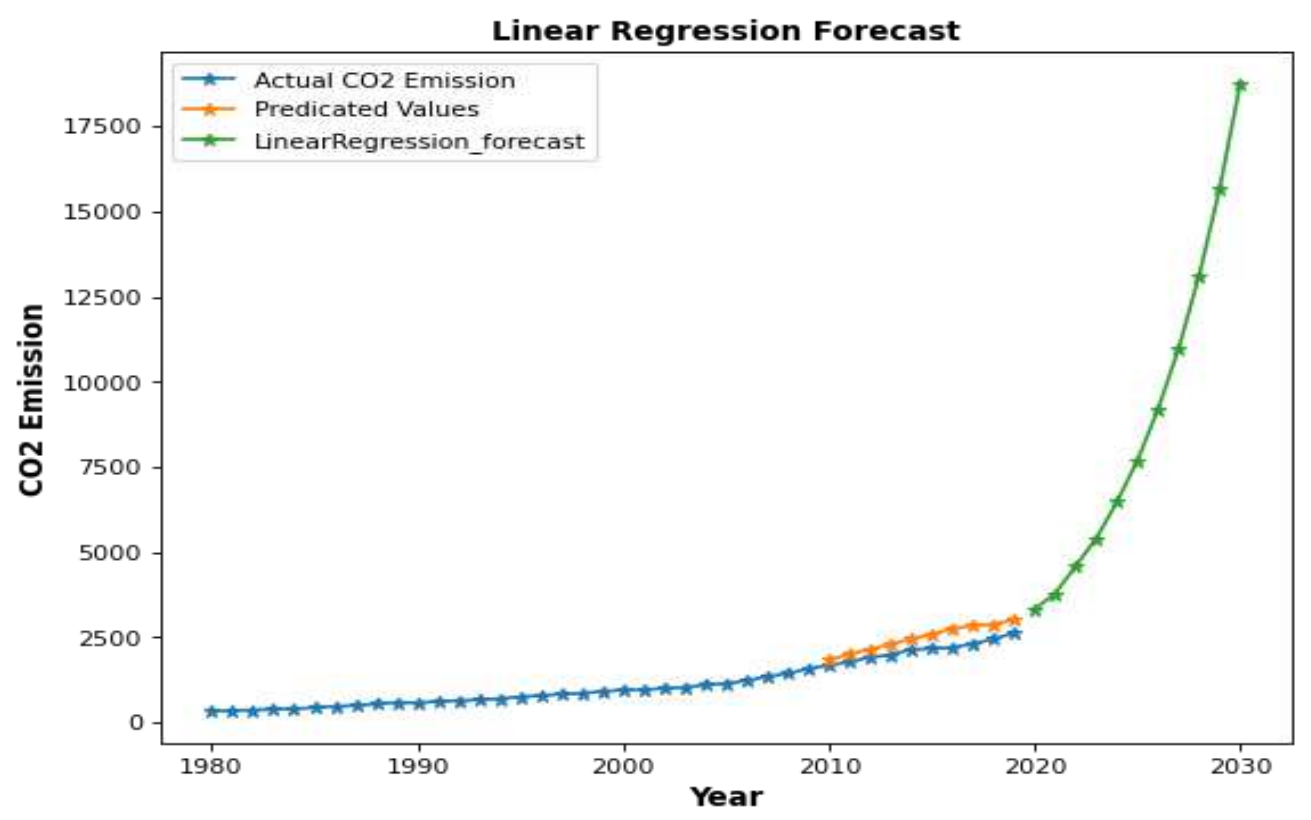

Figure 5. Linear Regression forecasting vs. Actual $\mathrm{CO}_{2}$ emissions 


\section{Random Forest Forecast}

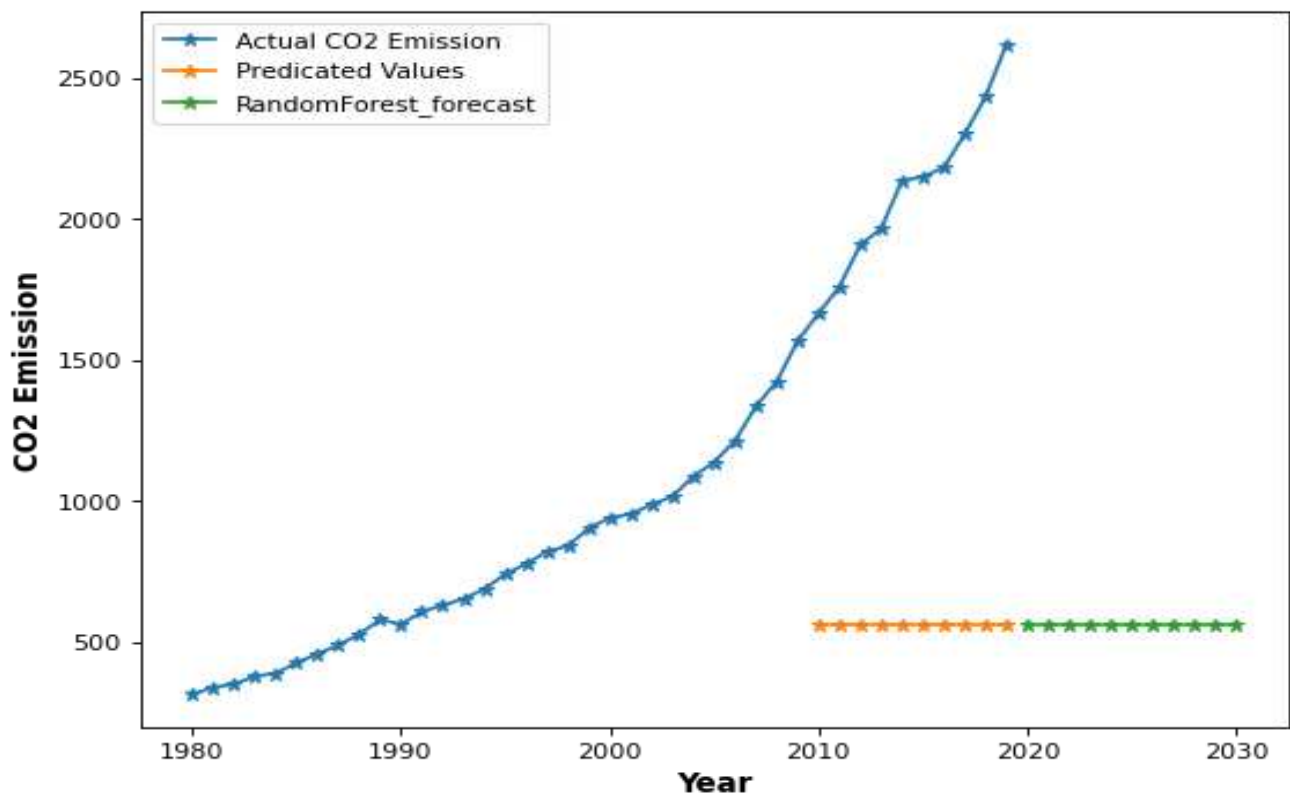

Figure 6. RF forecasting vs Actual $\mathrm{CO}_{2}$ emission

295 Figure 2, shows the $\mathrm{CO}_{2}$ predicted values which is quite different for Actual emissions. Therefore, we can conclude that 296 Random forest model is not appropriate for $\mathrm{CO}_{2}$ emissions forecasting.

\subsubsection{Holt-Winter model Forecasting}

298 We can see that Holt-Winter predicted values are quite similar to actual $\mathrm{CO}_{2}$ emissions. This model can also be considered 299 one of the effective forecasting models if we compromise a few performance metric values.

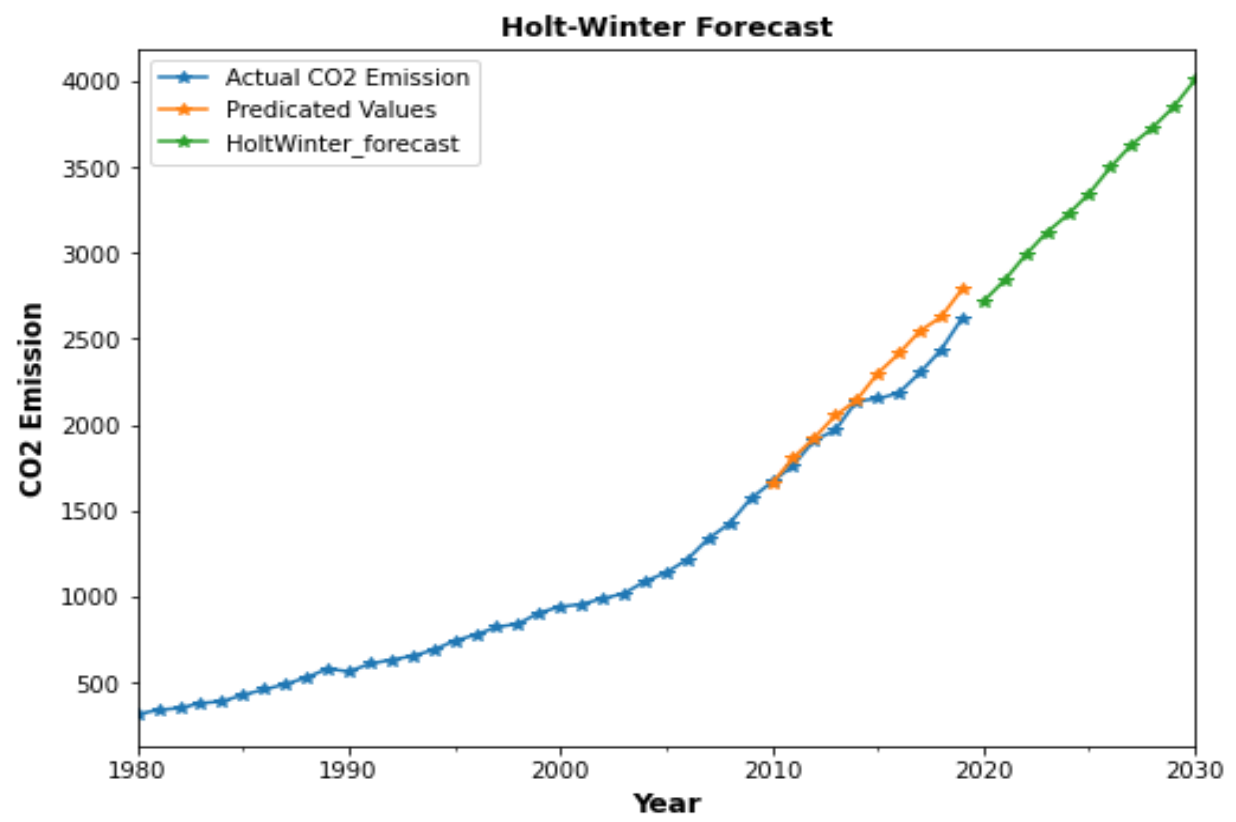

Figure 7. Holt-Winter forecasting vs. Actual $\mathrm{CO}_{2}$ emissions

\subsubsection{LSTM model Forecasting}

303 Figure 8, shows, LSTM behaviour for prediction and forecasting of the $\mathrm{CO}_{2}$ emissions. 


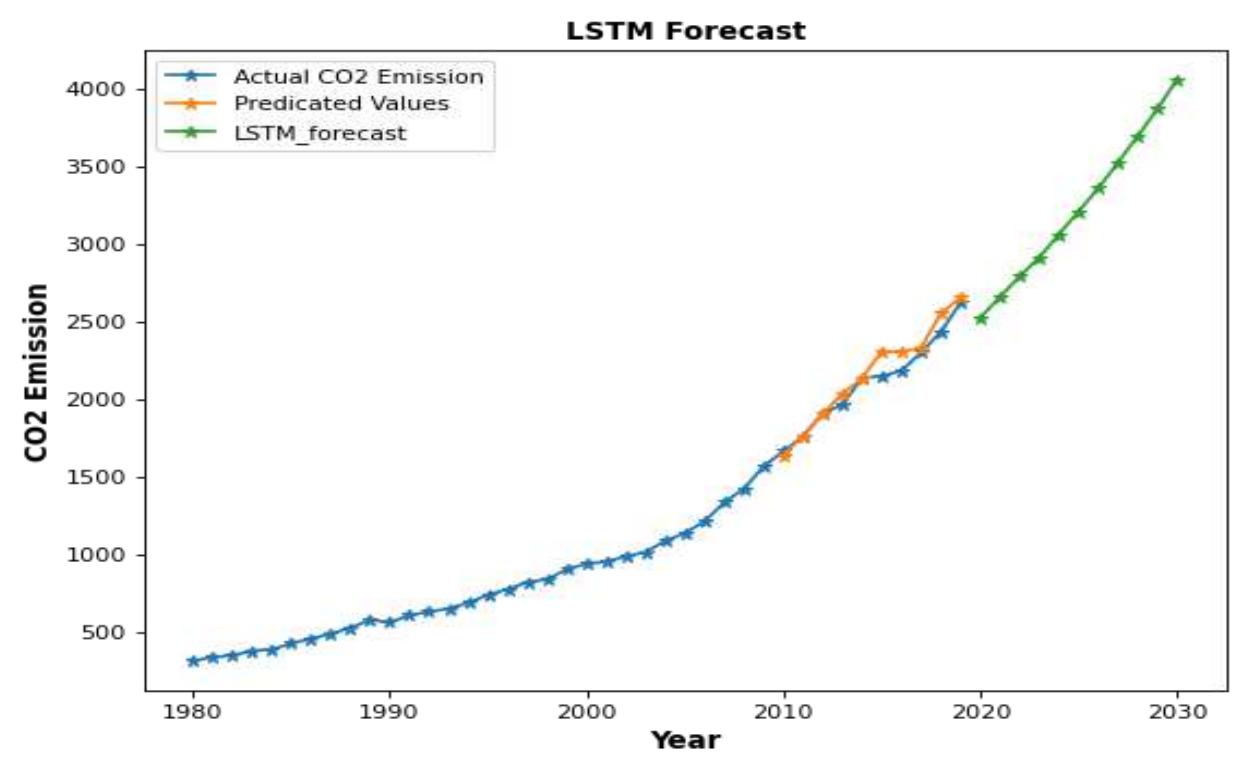

Figure 8. LSTM forecasting vs. Actual $\mathrm{CO}_{2}$ emissions

Observations from figure 8 shows that when we compare the actual $\mathrm{CO}_{2}$ emissions and predicted value (as indicated from 2010 to 2020), it is almost same. We have already analysed from figure 2, that LSTM is best performing model in terms

\subsection{Comparative Forecasting}

In this section, we have shown the comparative forecasting values for $\mathrm{CO}_{2}$ emissions from 2020 to 2030.

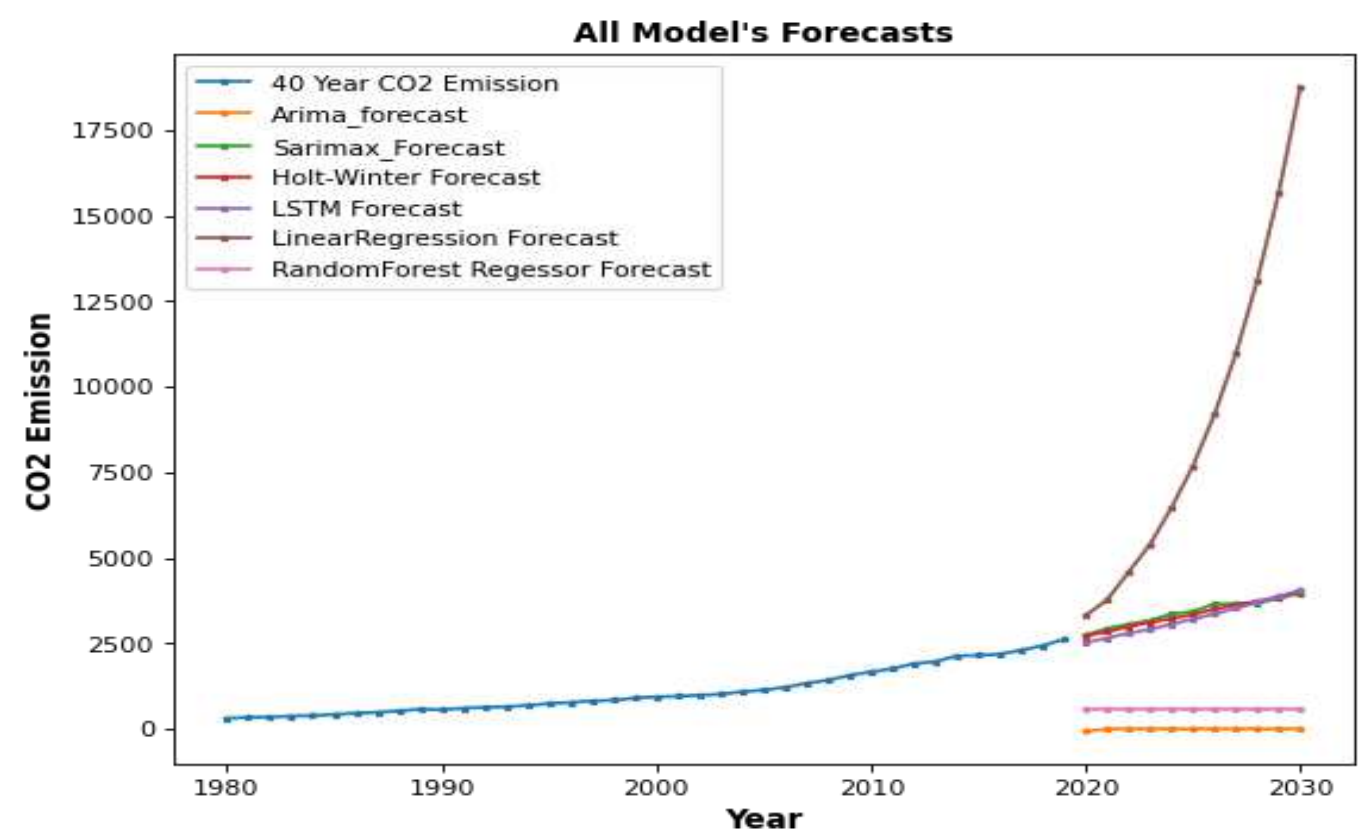

Figure 9 Comparative forecasting of $\mathrm{CO}_{2}$ emissions from the year 2020 to 2030

313 Observations from figure 9 show that LSTM, SARIMAX, and Holt-Winter are appropriate forecasting models. Further, when we look at the performance metric values, then LSTM is the best performing model for forecasting the $\mathrm{CO}_{2}$ emissions.

315 Here the contribution of this work, can also be seen as to control the $\mathrm{CO}_{2}$ emission to save human lives.

\section{Conclusion and Future Research Directions}


In this work, we have applied the machine learning, deep learning and statistical models to forecast the $\mathrm{CO}_{2}$ emissions in 318 India. For the same, we have considered nine performance metrics to evaluate the effectiveness of the applied models. 319 Observations from the results show that LSTM, SARIMAX, and Holt-Winter are better performing models to forecast $320 \mathrm{CO} 2$ emission.

To analyze the performance of the applied models, we have used the 40 years of $\mathrm{CO}_{2}$ emissions data. Section 6.2 .6 and performance metric values conclude that LSTM is the best forecasting model. Appropriate $\mathrm{CO}_{2}$ emission forecasting would be fruitful for the upcoming governments to frame their policies to abide by the UN-specific reduction measures, i.e. reducing $\mathrm{CO} 2$ emission by $58 \%$ by 2030 .

This work has not inculcated many factors such as the growth of population, advancing technology, switch to renewable energy sources, and future government actions. These are some exogenous factors that future researchers can explore. They may find a way to deal with such deviations due to these factors so that the new models could forecast data with more accurate $\mathrm{CO}_{2}$ emissions.

\section{Declarations}

Ethical Approval Not applicable

\section{Consent to Participate Not applicable}

Consent to Publish Permitted for publication as per journal guidelines

Funding Not applicable

\section{Authors' contributions}

Surbhi Kumari: Introduction, literature review, and performed the experiments.

Sunil Kumar Singh: conceptualised and designed the experiments and analyzed and interpreted the results.

Conflicts of interest/Competing interests There is no conflict of interest whatsoever with any other work by any of the authors of this work

Data Availability The data that support the findings of this study are openly available and it will be provided on request.

\section{References}

Abdullah, L., \& Pauzi, H. M. (2015). Methods in forecasting carbon dioxide emissions: A decade review. Jurnal Teknologi, $75(1)$.

Ahmed, N. K., Atiya, A. F., El Gayar, N., \& El-Shishiny, H. (2010). An empirical comparison of machine learning models for time series forecasting. Econometric Reviews, 29(5), 594-621. https://doi.org/10.1080/07474938.2010.481556

Amarpuri, L., Yadav, N., Kumar, G., \& Agrawal, S. (2019). Prediction of CO 2 emissions using deep learning hybrid approach: A Case Study in Indian Context. 2019 Twelfth International Conference on Contemporary Computing (IC3), 1-6.

Bonga, W. G., \& Chirowa, F. (2014). Level of Cooperativeness of Individuals to Issues of Energy Conservation. Available at SSRN 2412639.

Chatfield, C. (1978). The Holt-winters forecasting procedure. Journal of the Royal Statistical Society: Series C (Applied Statistics), 27(3), 264-279.

Crespo Cuaresma, J., Hlouskova, J., Kossmeier, S., \& Obersteiner, M. (2004). Forecasting electricity spot-prices using linear univariate time-series models. Applied Energy, 77(1), 87-106. https://doi.org/10.1016/S0306-2619(03)000965 
dos Santos, P. R. S., de Souza, L. B. M., Lélis, S. P. B. D., Ribeiro, H. B., Borges, F. A. S., Silva, R. R. V, Carvalho Filho, A. O., Araujo, F. H. D., Rabêlo, R. de A. L., \& Rodrigues, J. J. P. C. (2021). Prediction of COVID-19 using timesliding window: the case of Piauí state-Brazil. 2020 IEEE International Conference on E-Health Networking, Application \& Services (HEALTHCOM), 1-6.

Elsworth, S., \& Güttel, S. (2020). Time Series Forecasting Using LSTM Networks: A Symbolic Approach. http://arxiv.org/abs/2003.05672

Fang, X., Liu, W., Ai, J., He, M., Wu, Y., Shi, Y., Shen, W., \& Bao, C. (2020). Forecasting incidence of infectious diarrhea using random forest in Jiangsu Province, China. BMC Infectious Diseases, 20(1), 1-8.

Gopu, P., Panda, R. R., \& Nagwani, N. K. (2021). Time Series Analysis Using ARIMA Model for Air Pollution Prediction in Hyderabad City of India. In Soft Computing and Signal Processing (pp. 47-56). Springer.

Huang, C.-H., \& Hsieh, S.-H. (2020). Predicting BIM labor cost with random forest and simple linear regression. Automation in Construction, 118, 103280.

Kumar, R., Kumar, P., \& Kumar, Y. (2020). Time Series Data Prediction using IoT and Machine Learning Technique. Procedia Computer Science, 167, 373-381. https://doi.org/10.1016/j.procs.2020.03.240

Kumar, S., Mishra, S., \& Singh, S. K. (2020). A machine learning-based model to estimate PM2. 5 concentration levels in Delhi's atmosphere. Heliyon, 6(11), e05618.

Kumar, S., Mishra, S., \& Singh, S. K. (2021). Deep Transfer Learning-based COVID-19 prediction using Chest X-rays. Journal of Health Management, 23(4), 730-746.

Lepore, A., dos Reis, M. S., Palumbo, B., Rendall, R., \& Capezza, C. (2017). A comparison of advanced regression techniques for predicting ship CO2 emissions. Quality and Reliability Engineering International, 33(6), 1281-1292.

Masini, R. P., Medeiros, M. C., \& Mendes, E. F. (2021). Machine Learning Advances for Time Series Forecasting. Journal of Economic Surveys. https://doi.org/10.1111/joes.12429

Nontapa, C., Kesamoon, C., Kaewhawong, N., \& Intrapaiboon, P. (2020). A New Time Series Forecasting Using Decomposition Method with SARIMAX Model. International Conference on Neural Information Processing, 743751.

Nyoni, T., \& Bonga, W. G. (2019). Prediction of co2 emissions in india using arima models. DRJ-Journal of Economics \& Finance, 4(2), 1-10.

Özmen, E. S. (2021). Time Series Performance and Limitations with SARIMAX: An Application with Retail Store Data. Electronic Turkish Studies, 16(5).

Pino-Mejías, R., Pérez-Fargallo, A., Rubio-Bellido, C., \& Pulido-Arcas, J. A. (2017). Comparison of linear regression and artificial neural networks models to predict heating and cooling energy demand, energy consumption and $\mathrm{CO} 2$ emissions. Energy, 118, 24-36.

Sbrana, G. (2021). High-dimensional Holt-Winters trend model: Fast estimation and prediction. Journal of the Operational Research Society, 72(3), 701-713.

Shaikh, S., Gala, J., Jain, A., Advani, S., Jaidhara, S., \& Edinburgh, M. R. (2021). Analysis and Prediction of COVID-19 using Regression Models and Time Series Forecasting. 2021 11th International Conference on Cloud Computing, Data Science \& Engineering (Confluence), 989-995.

Wang, Q., Li, S., \& Pisarenko, Z. (2020). Modeling carbon emission trajectory of China, US and India. Journal of Cleaner Production, 258, 120723.

Wellington, G. (2019). Emissions in India using ARIMA Models 2 . Determine Stationarity of Time Series 4 . Diagnostic Checking 3 . Model Identification and Estimation 5 . Forecasting and Forecast Evaluation. Dynamic Research Journals, 4(2), 1-10.

Yin, L., \& Xie, J. (2021). Multi-temporal-spatial-scale temporal convolution network for short-term load forecasting of power systems. Applied Energy, 283, 116328.

Zuo, Z., Guo, H., \& Cheng, J. (2020). An LSTM-STRIPAT model analysis of China's 2030 CO2 emissions peak. Carbon Management, 11(6), 577-592. https://doi.org/10.1080/17583004.2020.1840869 